Supporting Information for

\title{
Exploring photogenerated molecular quartet states as spin qubits and qudits
}

\author{
Maximilian Mayländer, ${ }^{1}$ Su Chen, ${ }^{2}$ Emmaline R. Lorenzo, ${ }^{2}$ Michael R. Wasielewski, ${ }^{2 *}$ \\ Sabine Richert ${ }^{1 *}$ \\ ${ }^{1}$ Institute of Physical Chemistry, University of Freiburg, Albertstraße 21, 79104 Freiburg, Germany \\ 2 Department of Chemistry, Center for Molecular Quantum Transduction, and Institute for Sustainability \\ and Energy at Northwestern, Northwestern University, 2145 Sheridan Road, Evanston, \\ IL 60208-3113, United States \\ *E-mail: m-wasielewski@northwestern.edu, sabine.richert@physchem.uni-freiburg.de
}

\section{Table of Contents}

1 Synthesis and characterization of the PDI-TEMPO compounds S1

2 Variable temperature UV-vis spectroscopy $\quad$ S2

3 Additional fs-TA data and global kinetic analysis $\quad$ S2

4 Dark state EPR characterization $\quad$ S3

5 Additional transient EPR data $\quad$ S4

6 DFT calculations $\quad$ S14 


\section{List of Figures}

S1 Variable temperature UV-vis absorption spectra of PDI-TEMPO . . . . . . . . . . S2

S2 $\mathrm{fs}-\mathrm{TA}$ data of PDI-TEMPO in frozen solution . . . . . . . . . . . . . . . S3

S3 Global analysis of the fs-TA data of PDI-TEMPO in toluene . . . . . . . . . . . . . S3

S4 Continuous wave EPR spectrum and simulation . . . . . . . . . . . . . . . . . . . S S4

S5 Field-swept echo-detected EPR dark state spectra and simulation . . . . . . . . . . . . . . S4

S6 Determination of the nitroxide relaxation times . . . . . . . . . . . . . . . . S5

S7 Simulations of the transient cw EPR spectra of PDI-TEMPO . . . . . . . . . . . . S5

S8 Transient cw EPR spectra at the Q-band at various delays . . . . . . . . . . . . . . S6

S9 Contour plot of the Q-band transient cw EPR spectra . . . . . . . . . . . . . . . . S6

S10 Field-swept echo-detected spectrum with and without light $\ldots \ldots \ldots \ldots$. . . . . . . S7

S11 Spin polarized spectra at different times after laser excitation . . . . . . . . . . . . . . S8

S12 Subtraction of the ground state radical contribution from the spin polarized spectra . . . . S9

S13 Global analysis of the spin polarized signal as a function of time . . . . . . . . . . . . . . S9

S14 Transient nutation data at selected magnetic fields . . . . . . . . . . . . . . . . . S10

S15 Quartet state coherence times at different temperatures $\left(\left| \pm \frac{1}{2}\right\rangle \leftrightarrow\left| \pm \frac{3}{2}\right\rangle\right.$ transition) . . . . S11

S16 Quartet state coherence times at $80 \mathrm{~K}$ at different magnetic field positions . . . . . . . . . S11

S17 Determination of the quartet spin lattice relaxation time . . . . . . . . . . . . . S11

S18 Fourier transform of the Rabi oscillation time traces recorded at $80 \mathrm{~K} \ldots \ldots \ldots$. . . . . S12

S19 Rabi oscillation data acquired at $60 \mathrm{~K} \ldots \ldots \ldots \ldots \ldots$. . . . . . . . . . . . . . . . . .

S20 Rabi oscillation data at $80 \mathrm{~K}$ at different magnetic field positions . . . . . . . . . . . . . S13

S21 Temperature dependence of the quartet state signal . . . . . . . . . . . . . . . . . S14

S22 Calculated molecular structure and spin density of the triplet state . . . . . . . . . . . . S14

S23 Computed spin densities of the doublet and quartet states . . . . . . . . . . . . . S15 


\section{Synthesis and characterization of the PDI-TEMPO compounds}

All chemicals were purchased from Sigma-Aldrich, Inc. unless noted otherwise. Mass spectroscopy was performed using a Bruker Autoflex III MALDI-TOF. Purification was performed using silica gel from Sorbent Technologies (Atlanta, GA).

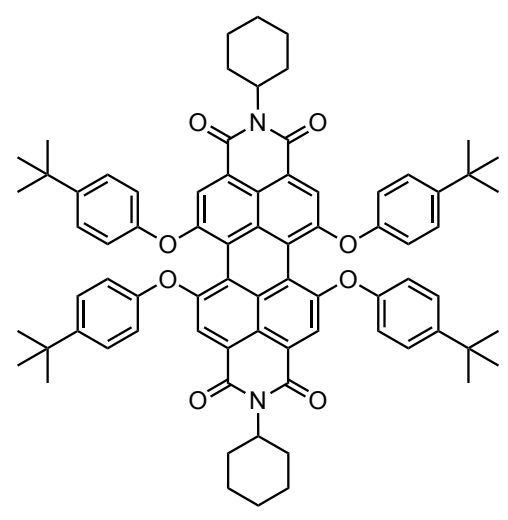

1

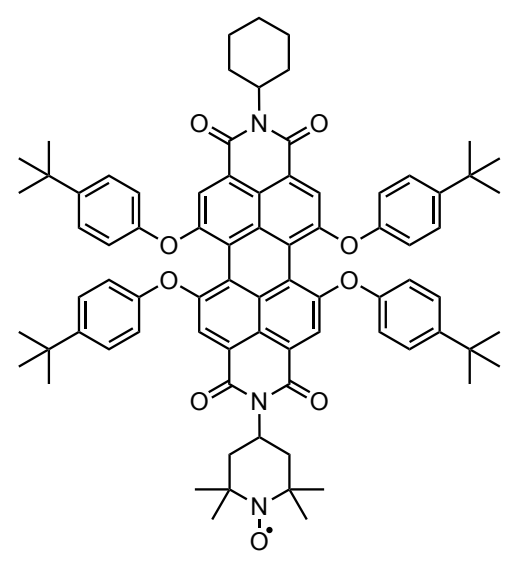

2

Compound 1 was synthesized according to literature procedures [1, 2].

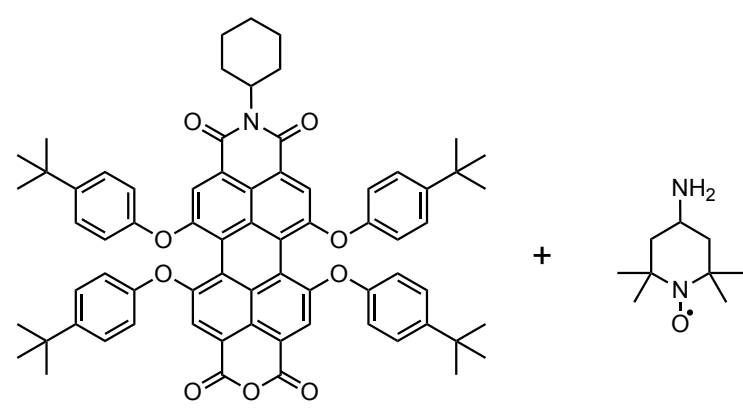

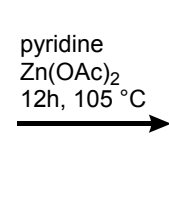

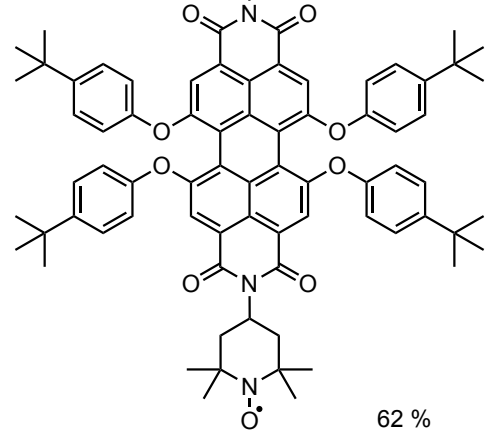

2

Compound a was synthesized according to the literature [1]. A $25 \mathrm{ml}$ two-necked bottle was charged with a stir bar, and compound a $(32 \mathrm{mg}, 0.03 \mathrm{mmol})$, 4-amino-TEMPO $(9 \mathrm{mg}, 0.05 \mathrm{mmol})$, pyridine $(2 \mathrm{ml})$, and $\mathrm{Zn}(\mathrm{OAc})_{2}(1 \mathrm{mg}, 0.005 \mathrm{mmol})$ were added. The two-necked bottle was then connected to a condenser with running water. The reaction was heated to $105^{\circ} \mathrm{C}$ and stirred for 12 hours. The solvent was then evaporated, and the product was purified by column chromatography (DCM as eluent). $22.6 \mathrm{mg}$ of 2 was obtained in $62 \%$ yield. MALDI-TOF: calculated for $[\mathrm{M}]^{-} \mathrm{m} / \mathrm{z}=1218.621$, found 1218.161 . Rf in DCM 0.3 . 


\section{Variable temperature UV-vis spectroscopy}

To characterize the absorption spectrum of PDI-TEMPO at low temperatures, as applied in the EPR experiments, variable temperature UV-vis absorption spectroscopy was performed on a sample of PDI-TEMPO in 2-methyltetrahydrofuran using an optical cryostat. Due to excitation light scattering and condensation of residual moisture inside the cryostat, a polynomial background-correction needed to be applied to the spectra recorded at low temperatures. As can be seen from Figure S1, no signs of aggregation could be perceived when the temperature was gradually lowered from room temperature to $100 \mathrm{~K}$. As expected, the individual absorption bands become narrower when the sample is cooled down, accompanied by a slight increase in absorbance.

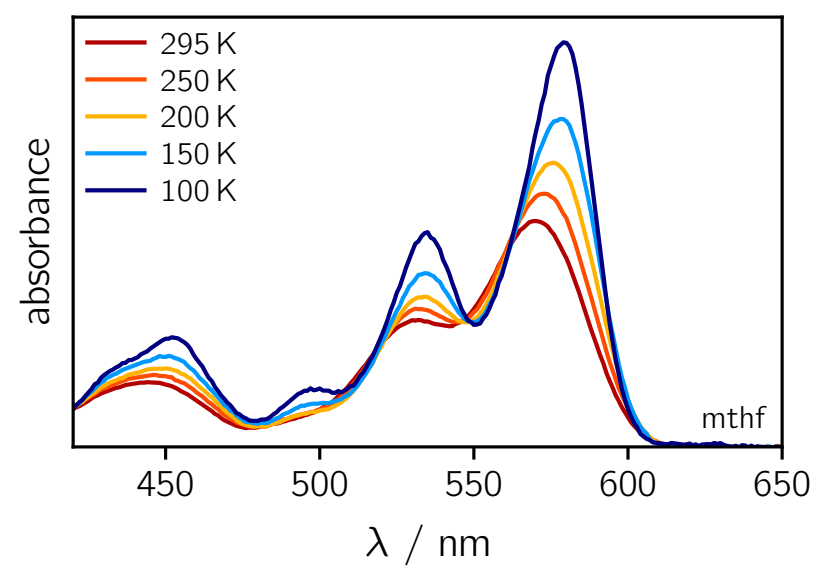

Figure S1: Variable temperature (as indicated) UV-vis absorption spectra of PDI-TEMPO dissolved in 2methyltetrahydrofuran recorded in a $1 \mathrm{~mm}$ cuvette.

\section{Additional fs-TA data and global kinetic analysis}

To verify whether the excited state dynamics differ under the conditions applied in the EPR measurements, femtosecond TA experiments were also performed in frozen 2-methyltetrahydrofuran solution at $85 \mathrm{~K}$ using an optical cryostat. The solvent, 2-methyltetrahydrofuran, was distilled, deoxygenated, and stored in the glove box where the sample solutions were prepared. The sample cell, composed of two quartz windows separated by a teflon spacer $(\sim 2 \mathrm{~mm})$, was filled with the solution and assembled under oxygen exclusion in the glove box. The sample concentration was adjusted to yield an OD of about 0.3-0.6 at the excitation wavelength in the sample cuvette and the data are shown in Figure S2. As can be seen from the figure, EISC also takes place in frozen solution, however at a slower rate (time constant of roughly $300 \mathrm{ps}$ ).

Several scans needed to be averaged until an acceptable signal-to-noise ratio could be obtained. Between individual scans, the cryostat was laterally displaced, whenever necessary, to minimize the effects of sample degradation on the acquired spectra. All TA spectra were acquired using an excitation energy of $\sim 1 \mu \mathrm{J}$. Artefacts from excitation light scattering at the excitation wavelength could not be avoided when using the cryostat. The wavelength ranges dominated by such artefacts were cut out from the spectra. The data were background-corrected and chirp-corrected using home-written MATLAB routines.

To get a better estimate of the time constants of the excited state reaction processes, a global kinetic analysis of the recorded fs-TA data was carried out. The species associated spectra obtained for PDI-TEMPO in toluene from target analysis assuming a sequential model are shown below (cf. Figure S3). 

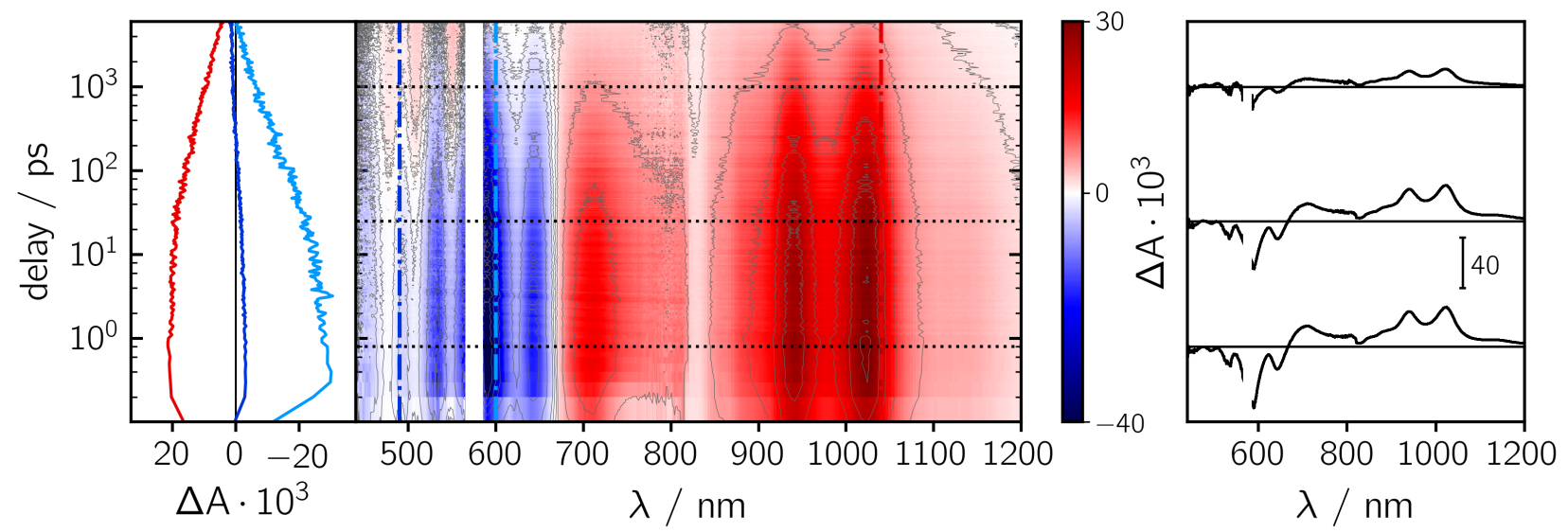

Figure S2: Femtosecond transient absorption data of PDI-TEMPO recorded in frozen 2-methyltetrahydrofuran solution at $85 \mathrm{~K}$ after photoexcitation at $575 \mathrm{~nm}$. The wavelength ranges dominated by artefacts from excitation light scattering were cut out from the spectra.
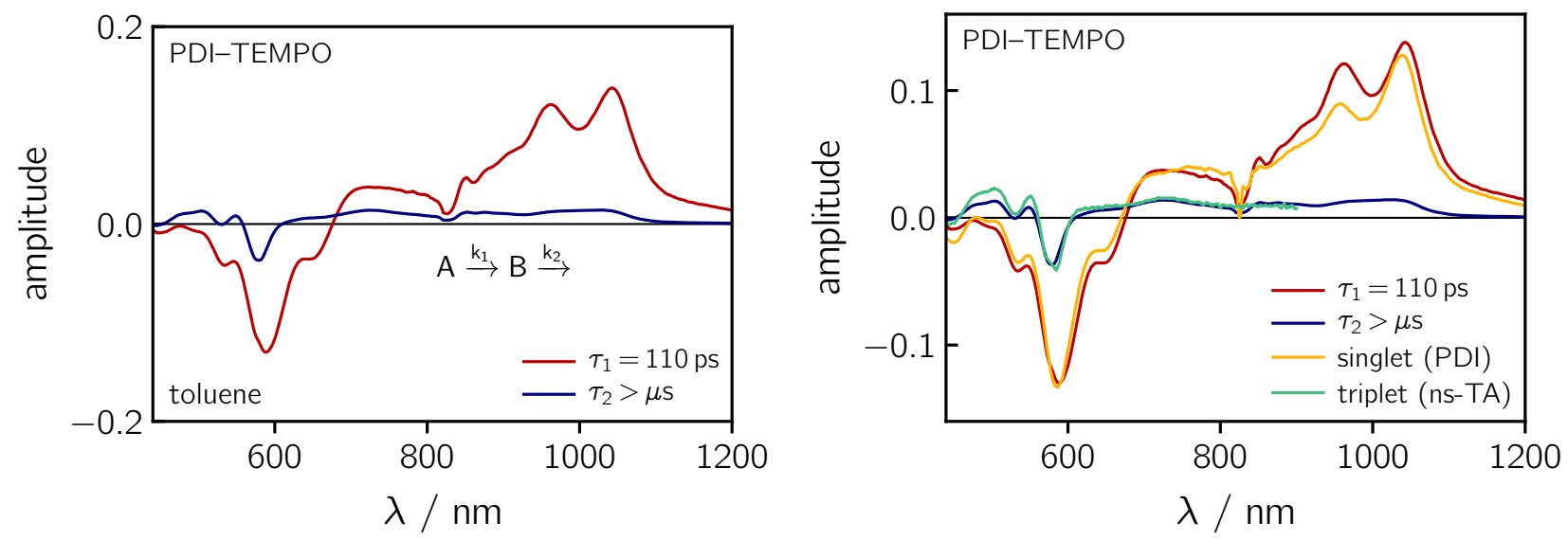

Figure S3: Species associated spectra (SAS) and time constants obtained from a target analysis of the fs-TA spectra of PDI-TEMPO in toluene at room temperature (left) and comparison of the SAS obtained for PDI-TEMPO with the scaled fs-TA spectrum of PDI (pure excited singlet state) and a ns-TA spectrum of PDI-TEMPO (triplet state) (right).

At room temperature, the excited state kinetics of PDI-TEMPO in toluene could be satisfactorily described with two time constants. The first time constant of $110 \mu \mathrm{s}$ corresponds to the time constant of enhanced intersystem crossing to the triplet state, whereas the second time constant $(>\mu \mathrm{s})$ is attributed to the decay of the excited triplet state as could be confirmed by comparison of the spectral signatures with a spectrum acquired on a ns-TA setup (cf. Figure S3, right).

\section{Dark state EPR characterization}

To characterize the g-tensor and nitrogen hyperfine couplings of the TEMPO radical, a continuous wave EPR spectrum of PDI-TEMPO in toluene was recorded in the dark at room temperature.

For an accurate determination of the g-tensor, the cw EPR spectrum was fit together with the field-swept echo-detected spectra of PDI-TEMPO recorded at $80 \mathrm{~K}$ (frozen toluene) at X-and Q-band microwave frequencies on a Bruker ELEXSYS E580 spectrometer. The simulation was carried out in MATLAB using EasySpin [3] functions in combination with custom-written fitting routines. As a result from this 'global fit' a g-tensor of [ 2.01002 .00642 .0021$]\left(g_{\text {iso }}=2.0062\right)$ and $\mathbf{A}$-tensor of $\mathbf{A}_{\mathbf{N}}=\left[\begin{array}{ll}19 & 95\end{array}\right] \mathrm{MHz}$ was obtained for the ground state nitroxide radical. The corresponding experimental data and result of the 'global' simulation 
are shown below in Figures S4 and S5.

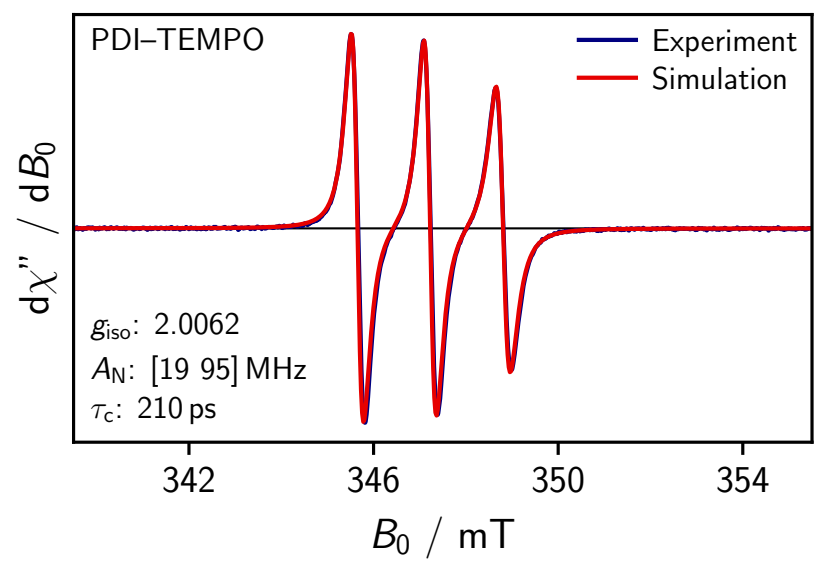

Figure S4: Continuous wave EPR spectrum of PDI-TEMPO recorded in toluene solution at room temperature using a modulation amplitude of $0.1 \mathrm{mT}$ together with the corresponding simulation from a global fit of the $\mathbf{g}$ and $\mathbf{A}_{\mathbf{N}}$-tensors as described in the text.
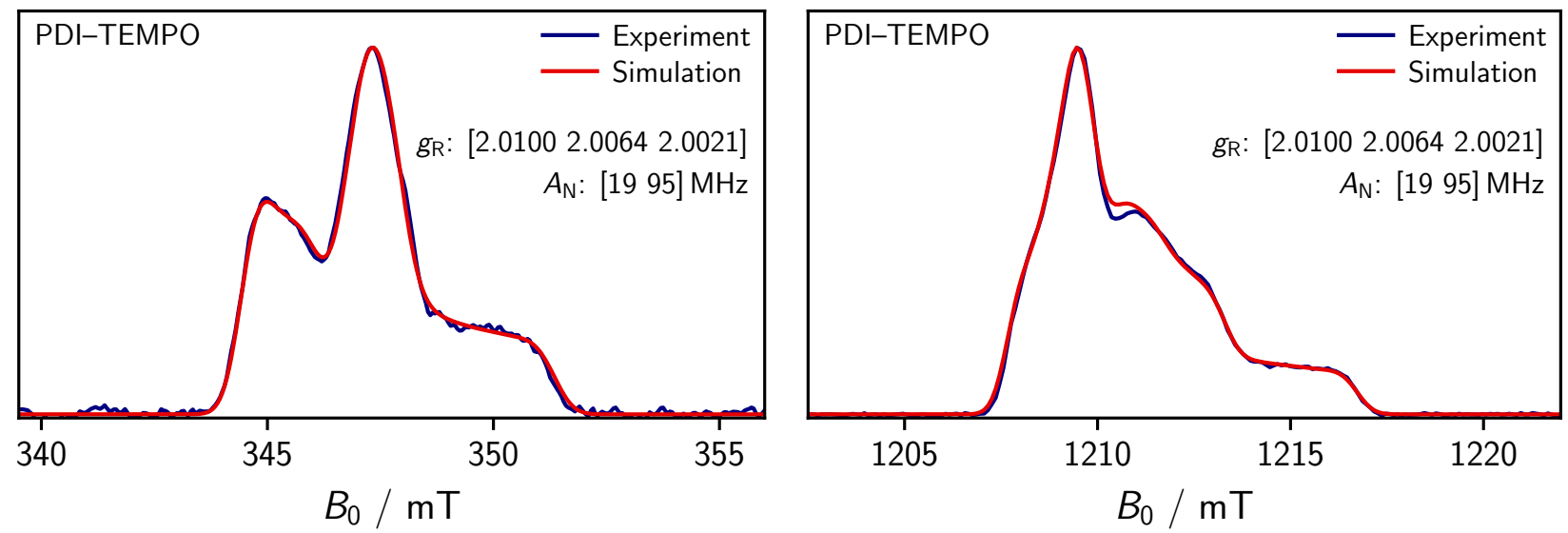

Figure S5: Field-swept echo-detected EPR spectrum of PDI-TEMPO recorded in the dark at the X-band (left) and Q-band (right) in frozen toluene at $80 \mathrm{~K}$ together with the result of a global simulation of the data using the simulation parameters indicated in the figure.

As can be seen from Figure S4, the cw EPR spectrum of PDI-TEMPO is not isotropic at room temperature. A rotational correlation time of $210 \mathrm{ps}$ was obtained from the simulation.

To characterize the relaxation behavior of PDI-TEMPO, the spin memory and spin lattice relaxation times of the nitroxide were determined in the dark at $80 \mathrm{~K}$. A fit of the phase memory time yielded about $2 \mu \mathrm{s}$ for the nitroxide in the dark at $80 \mathrm{~K}$. $T_{1}$ was measured using the Picket-fence method (saturation recovery). As shown in Figure S6, a $T_{1}$ value of $\sim 0.2 \mathrm{~ms}$ was obtained by a fit of the data assuming a monoexponential decay. The $T_{1}$ relaxation behavior was found to be similar within the $x y$-region of the nitroxide spectrum and also does not change markedly with microwave frequency.

\section{Additional transient EPR data}

Numerical simulations of the transient cw EPR spectra obtained for PDI-TEMPO at the X-and Q-band are shown in Figure S7. The corresponding experimental spectra, recorded at $\sim 1 \mu$ s after laser excitation, are shown in the background in light gray. From the figure it can be seen that the net polarization of the 

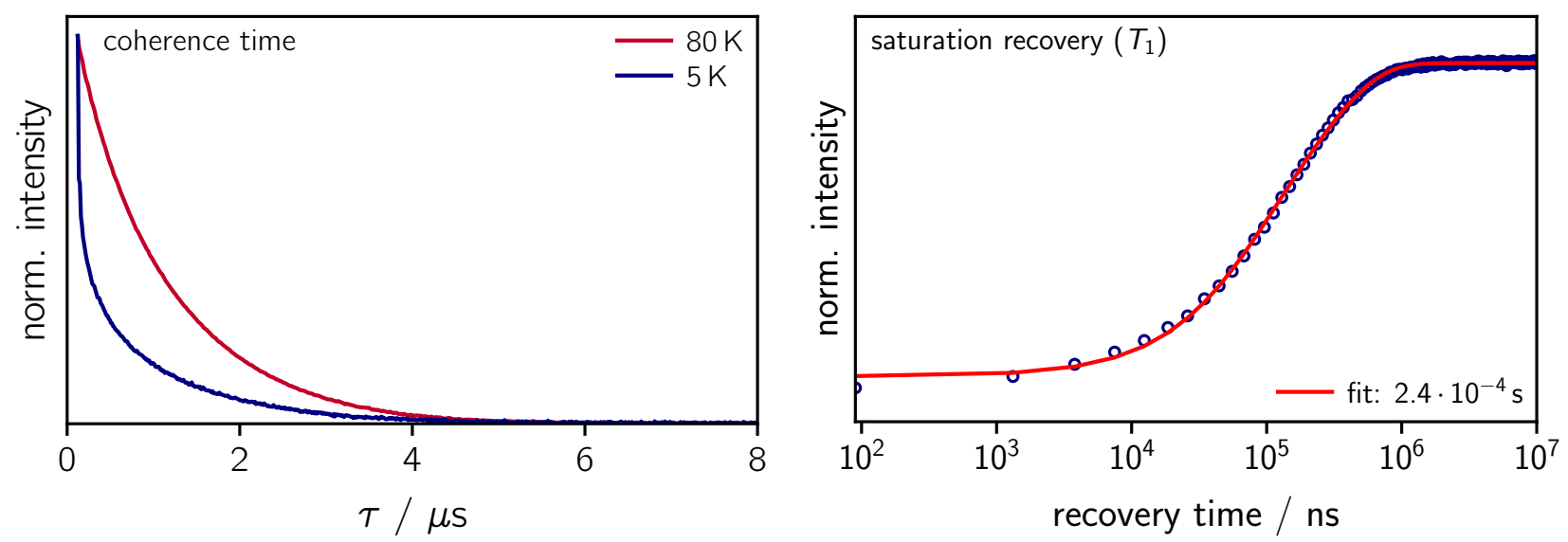

Figure S6: Measurement of the phase memory $\left(T_{\mathrm{m}}\right.$, left $)$ and spin-lattice $\left(T_{1}\right.$, right $)$ relaxation times of the nitroxide of PDI-TEMPO recorded at the Q-band in the dark.

quartet state is inverted between $\mathrm{X}$ - and Q-band, while the multiplet polarization remains similar and is characteristic of an overpopulation of the high field quartet $\pm \frac{1}{2}$ sublevels.
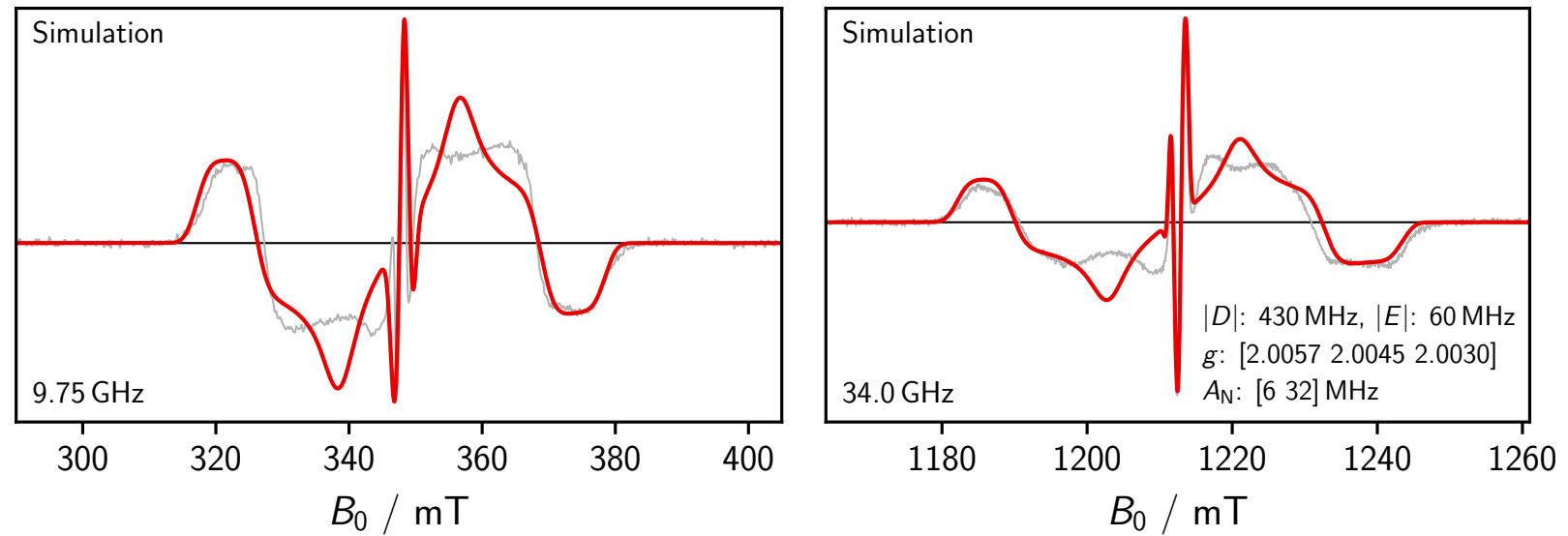

Figure S7: Simulations of the transient cw EPR spectra of PDI-TEMPO recorded at $80 \mathrm{~K}$ in frozen toluene at the $\mathrm{X}$ - (left) and Q-band (right). The simulation parameters are indicated in the figure.

The spectra for X-and Q-band were simulated with the same parameters (except for the linewidths and quartet high field populations), as indicated in the figure above. The g-tensor was calculated from the nitroxide g-tensor and a PDI triplet $g$-value of 2.0035 and kept fixed in the simulation. The nitrogen hyperfine couplings expected for the quartet state were calculated from $\frac{1}{3} A_{N}$ (TEMPO) and were also assumed to be invariant. Only the high field quartet state populations were adapted until the best match was obtained for the following population vectors (low to high energy) $p=\left[\begin{array}{lllllllllllll}0 & 0 & 0 & 0.10 & 0.27 & 0.13 & 0.15 & 0.20\end{array}\right.$

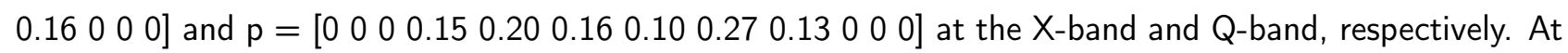
the X-band, the populations of the quartet $\left| \pm \frac{1}{2}\right\rangle$ sublevels are thus inverted compared to the situation at Q-band frequencies, with might be explained by a level crossing.

Figure S8 shows the Q-band transient cw EPR spectra of PDI-TEMPO at various different times after laser excitation (as indicated). It is observed that the ratio of net to multiplet polarization decreases with time and that the whole spectrum inverts at times $>60 \mu \mathrm{s}$. This can also be seen more clearly in the contour plot in Figure S9. 


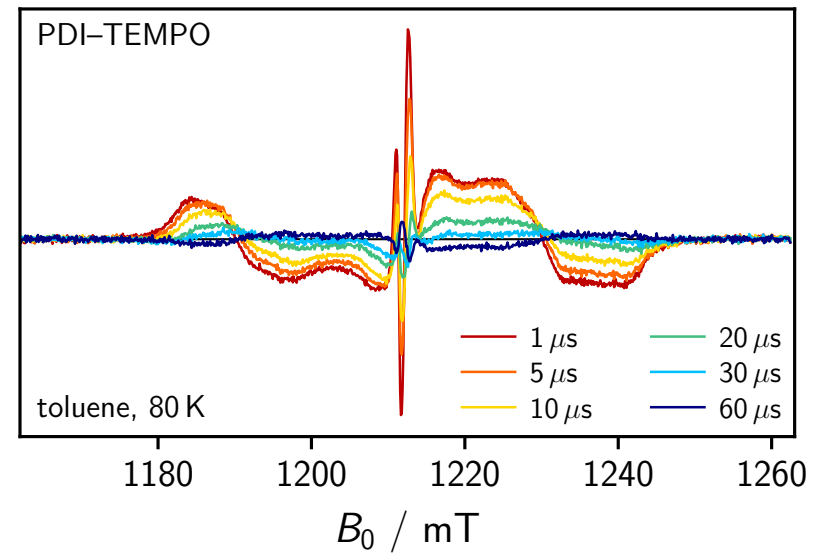

Figure S8: Transient cw EPR spectra of PDI-TEMPO in frozen toluene at various time delays after laser excitation (as indicated) recorded at the Q-band at $80 \mathrm{~K}$.

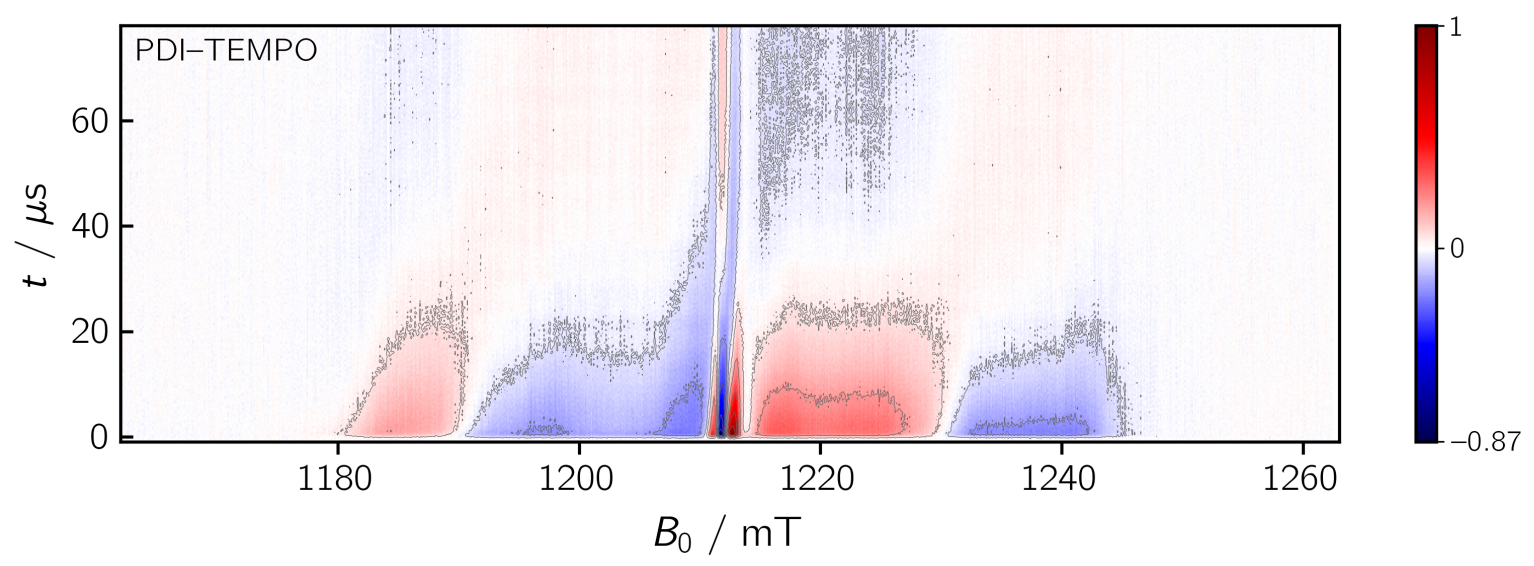

Figure S9: Contour plot of the Q-band transient cw EPR spectra of PDI-TEMPO in frozen toluene (80 K) as a function of time after laser excitation. 


\section{Echo-detected field sweep}

Figure S10 shows the field-swept echo-detected EPR spectrum recorded for PDI-TEMPO in frozen toluene at the Q-band with and without photoexcitation at $535 \mathrm{~nm}(\mathrm{DAF}=9 \mu \mathrm{s})$. The spectra were recorded at a microwave attenuation optimized for the quartet state, using identical settings (i.e. same video gain).

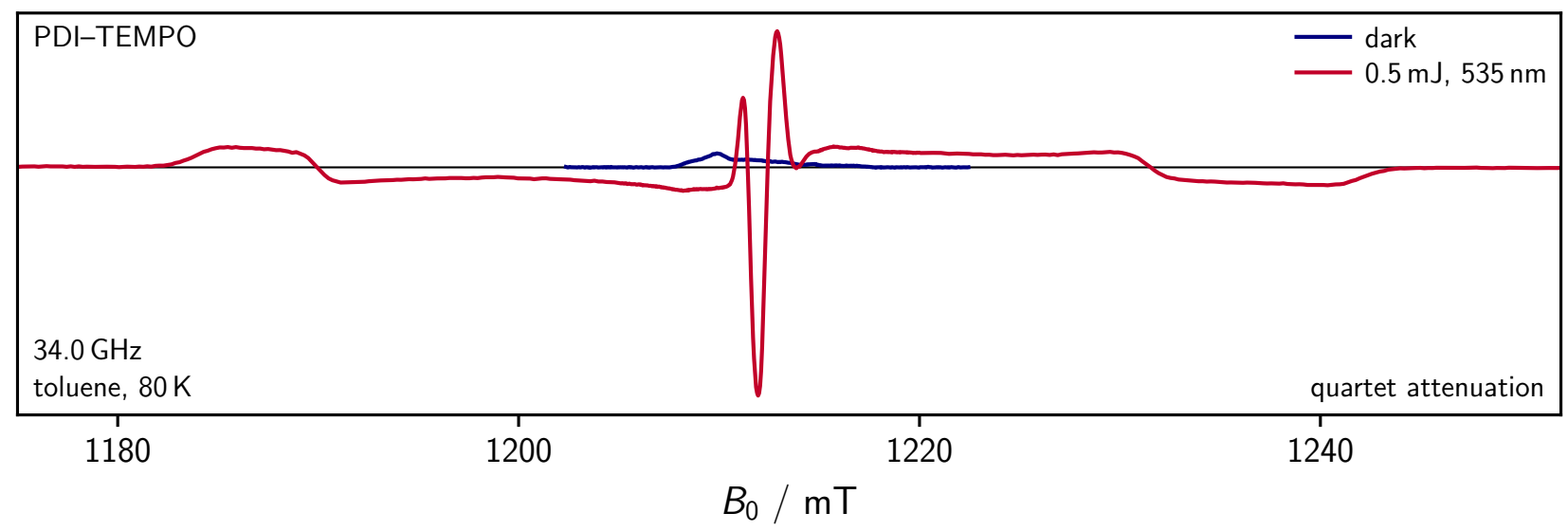

Figure S10: Field-swept echo-detected EPR spectrum of PDI-TEMPO recorded with and without photoexcitation at $535 \mathrm{~nm}$ at a DAF of $9 \mu \mathrm{s}$ and a microwave attenuation optimized for the quartet state.

\section{Spin polarization lifetime}

In Figure S11, the light-induced signal is shown at different time delays after photoexcitation (i.e. DAF).

The individual traces represent slices of the map shown in the main text. The dark signal before time zero was subtracted from the dataset. However, since the radical contribution necessarily diminishes after photoexcitation (as part of the sample contributes to quartet state formation), a contribution exhibiting the shape of a negatively polarized radical ground state (having the same kinetics as the quartet state) is observed in the spectra (reminiscent of a 'ground state bleach' in transient absorption spectroscopy). This contribution was subtracted from the data shown in Figure S11 and the main text to obtain the correct spectral shape of the quartet state.

Figure S12 illustrates the two spectral components and their contribution to the measured spectra at two different time delays.

Figure S13 shows the results of a global kinetic analysis of the polarization decay, assuming a sequential model (as indicated in the figure). It can be seen that the spectral features of the quartet state shift slightly and later invert on a time scale of roughly $50 \mu \mathrm{s}$. The inverted quartet signal has a very low intensity but persists for longer than $100 \mu \mathrm{s}$. Over time, the ratio of net to multiplet polarization diminishes. 

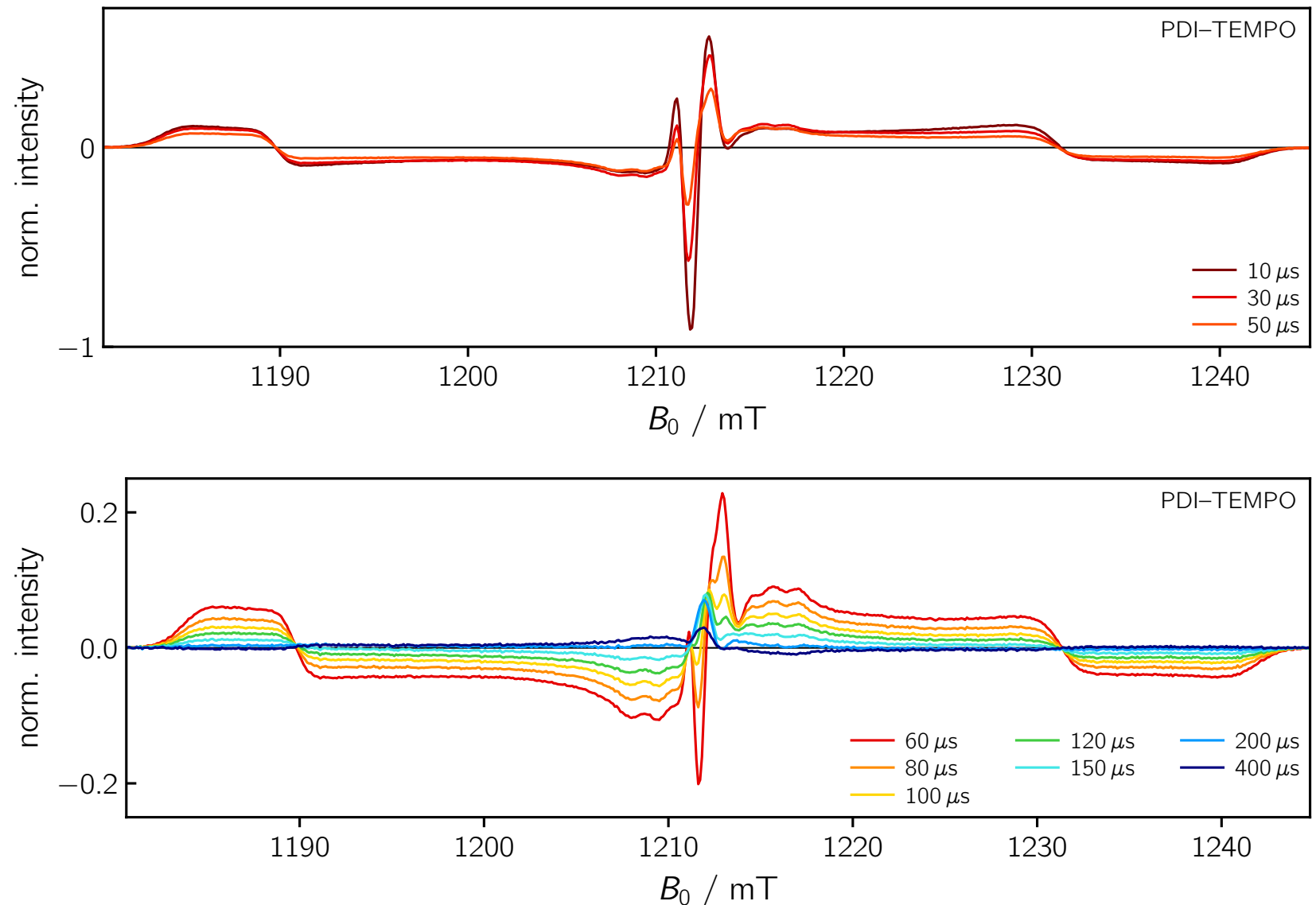

Figure S11: Spin polarized spectra of PDI-TEMPO shown at different time delays after photoexcitation (DAF, as indicated). The spectra represent individual slices of the map shown in the main text and were corrected for a residual nitroxide radical contribution after subtraction of the dark signal before time zero. 

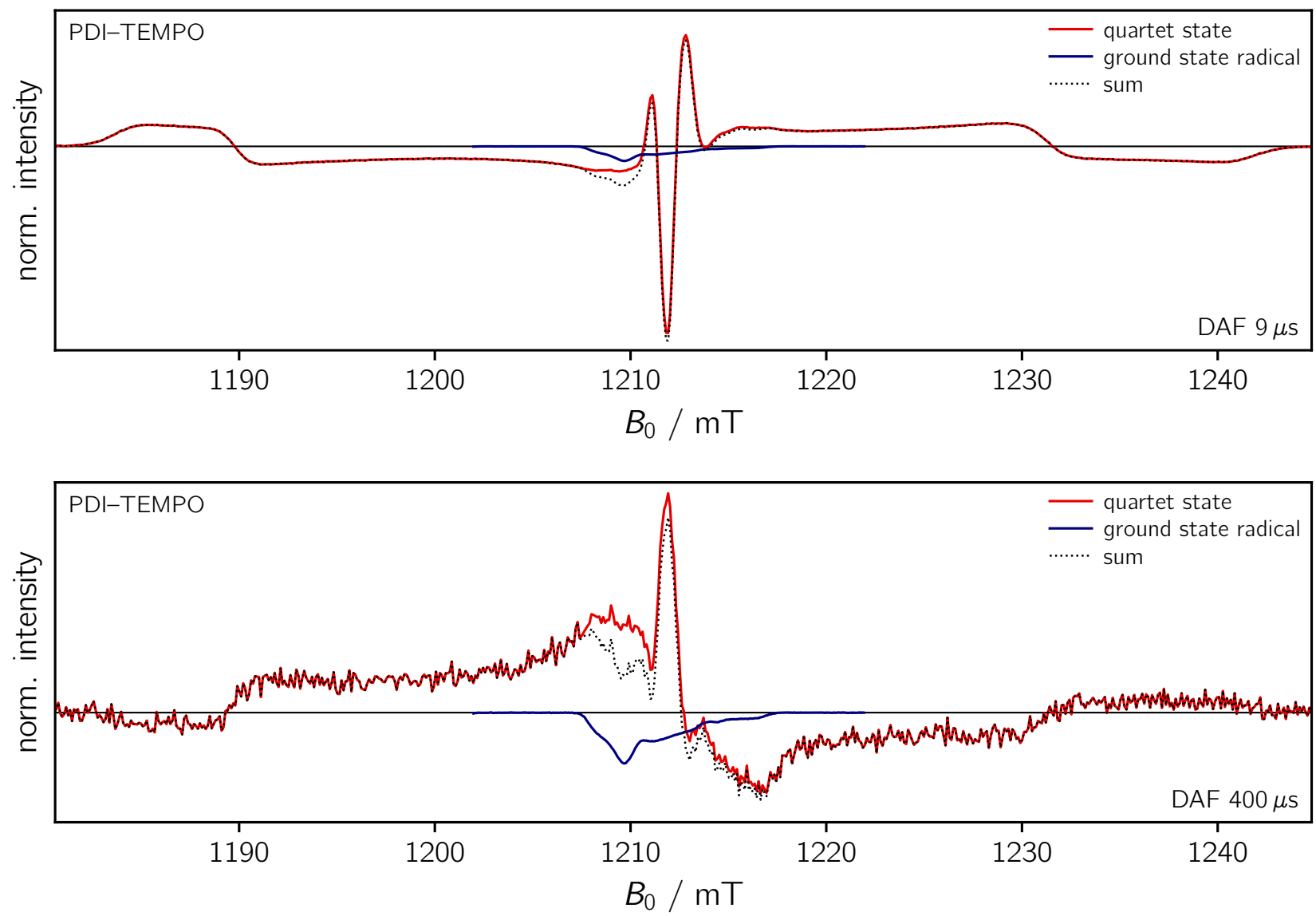

Figure S12: Illustration of the subtraction of the ground state radical contribution from the spin polarized spectra of PDI-TEMPO at two different DAF values of $9 \mu \mathrm{s}($ top) and $400 \mu \mathrm{s}$ (bottom) to obtain the spectral shape of the quartet state. The sum spectrum corresponds to the spectrum as measured in the experiment (after subtraction of the dark state radical signal before photoexcitation).

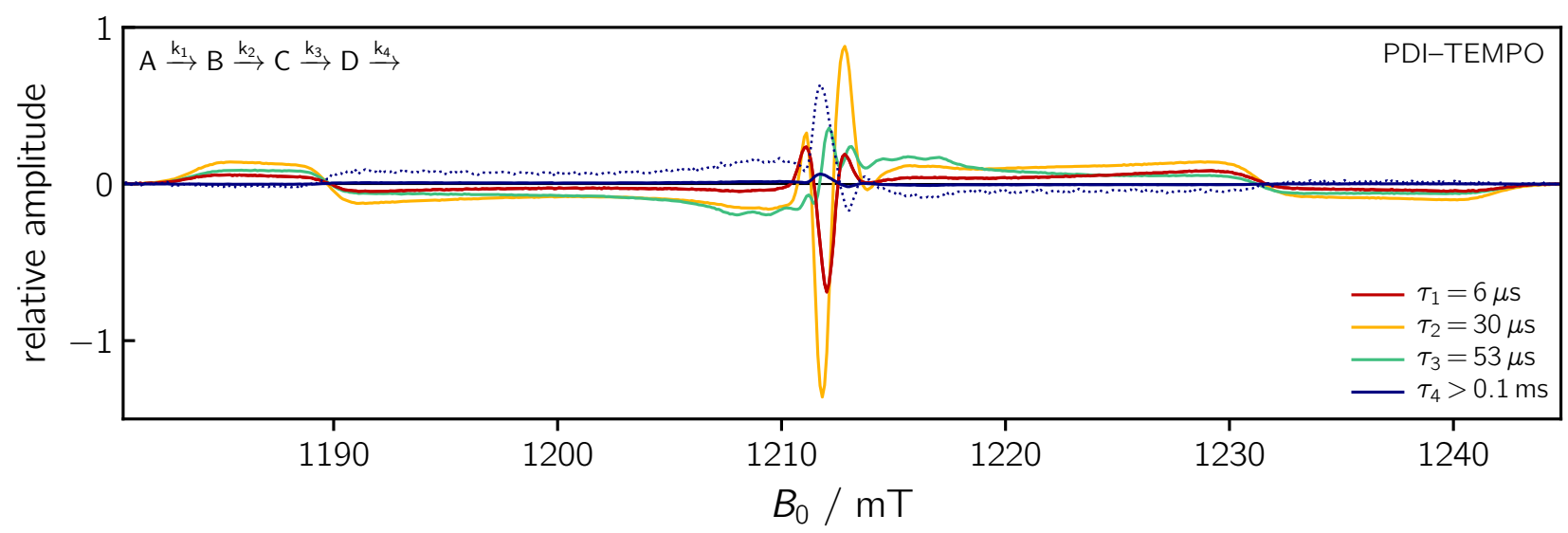

Figure S13: Global analysis of the spin polarized signal as a function of time after laser excitation. The species associated spectra are shown together with the obtained time constants and the kinetic model used in the simulation. The blue dotted line is a ten-fold amplification of the amplitude spectrum shown in blue. 


\section{Transient nutations}

For a better illustration of the obtained nutation frequencies, Figure S14 shows slices of the map shown in the main text at three different magnetic fields (same color code). The frequencies were normalized using the data obtained under the same conditions for PDI-TEMPO in the dark (pure doublet state). After photoexcitation, part of the radical signal persists and contributes to the transient nutation signal in the central region of the quartet spectrum (particularly, of course, at the field positions where the quartet signal crosses the zero line). Consequently, a doublet contribution is observed in addition to the expected nutation frequencies for the quartet state. In the region of the multiplet polarization $\left(\left| \pm \frac{1}{2}\right\rangle \leftrightarrow\left| \pm \frac{3}{2}\right\rangle\right.$ transitions $)$ the expected frequency amounts to $\sqrt{3} \omega_{R}$, while in the spectral region of the net polarization $\left(\left|+\frac{1}{2}\right\rangle \leftrightarrow\left|-\frac{1}{2}\right\rangle\right.$ transition) a nutation frequency of $2 \omega_{R}$ would be expected. $\omega_{R}$ is the reference frequency measured for the ground state radical in the dark (doublet multiplicity).

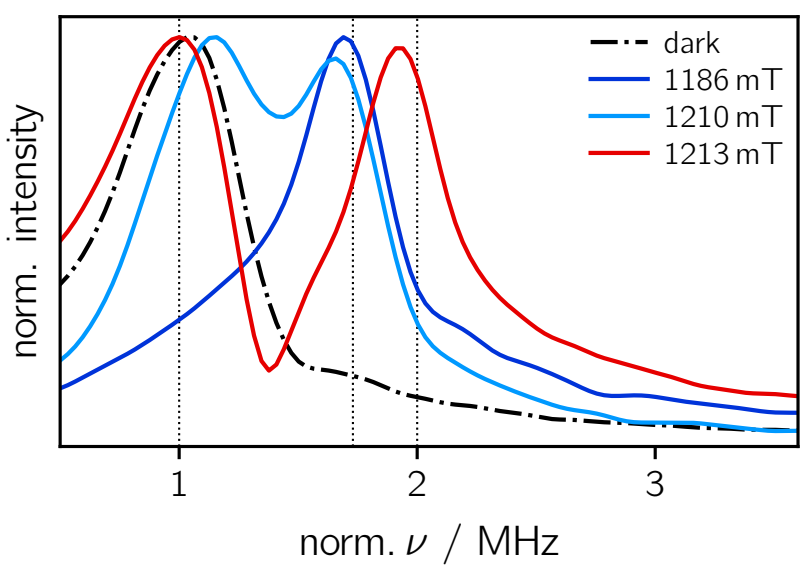

Figure S14: Transient nutation data shown at selected magnetic fields (as indicated) recorded at $80 \mathrm{~K}$ at the Q-band for PDI-TEMPO at DAF $=9 \mu \mathrm{s}$. The nutation frequency was normalized using that obtained for the nitroxide radical in the dark. The expected nutation frequencies are indicated by vertical dotted lines.

\section{Relaxation data}

Spin coherence times $\left(T_{\mathrm{m}}\right)$ of the quartet state were also measured at different temperatures and field positions. Figure S15 shows the corresponding data acquired at different temperatures at a magnetic field position corresponding to the wings of the quartet spectrum around $1230 \mathrm{mT}(34 \mathrm{GHz})$. When assuming a monoexponential (or biexponential) decay of the form

$$
I(\tau)=A \exp \left(-\frac{2 \tau}{T_{\mathrm{m}}}\right)
$$

time constants of approximately $0.4,0.7,1.1,1.6$, and $3.4 \mu$ s were obtained at $100 \mathrm{~K}, 80 \mathrm{~K}, 60 \mathrm{~K}, 40 \mathrm{~K}$ and $5 \mathrm{~K}$, respectively, whereas values of $0.8,1.8,2.0,2.2$, and $2.0 \mu \mathrm{s}$ were obtained at these temperatures in the center of the spectrum $\left(\left|+\frac{1}{2}\right\rangle \leftrightarrow\left|-\frac{1}{2}\right\rangle\right.$ transition, data shown in the main text).

The coherence times associated with the $\left|+\frac{1}{2}\right\rangle \leftrightarrow\left|-\frac{1}{2}\right\rangle$ transition were found to differ substantially compared to those measured at field positions corresponding to the $\left| \pm \frac{1}{2}\right\rangle \leftrightarrow\left| \pm \frac{3}{2}\right\rangle$ transition, while the differences within a particular transition (either $\left| \pm \frac{1}{2}\right\rangle \leftrightarrow\left| \pm \frac{3}{2}\right\rangle$ or $\left|+\frac{1}{2}\right\rangle \leftrightarrow\left|-\frac{1}{2}\right\rangle$ ) are much less pronounced.

Figure S16 shows the $T_{\mathrm{m}}$ data acquired at $80 \mathrm{~K}$ at different magnetic field positions within the quartet state spectrum, as indicated by the colored vertical lines in the corresponding field-swept echo-detected spectrum (Figure S16, left). 

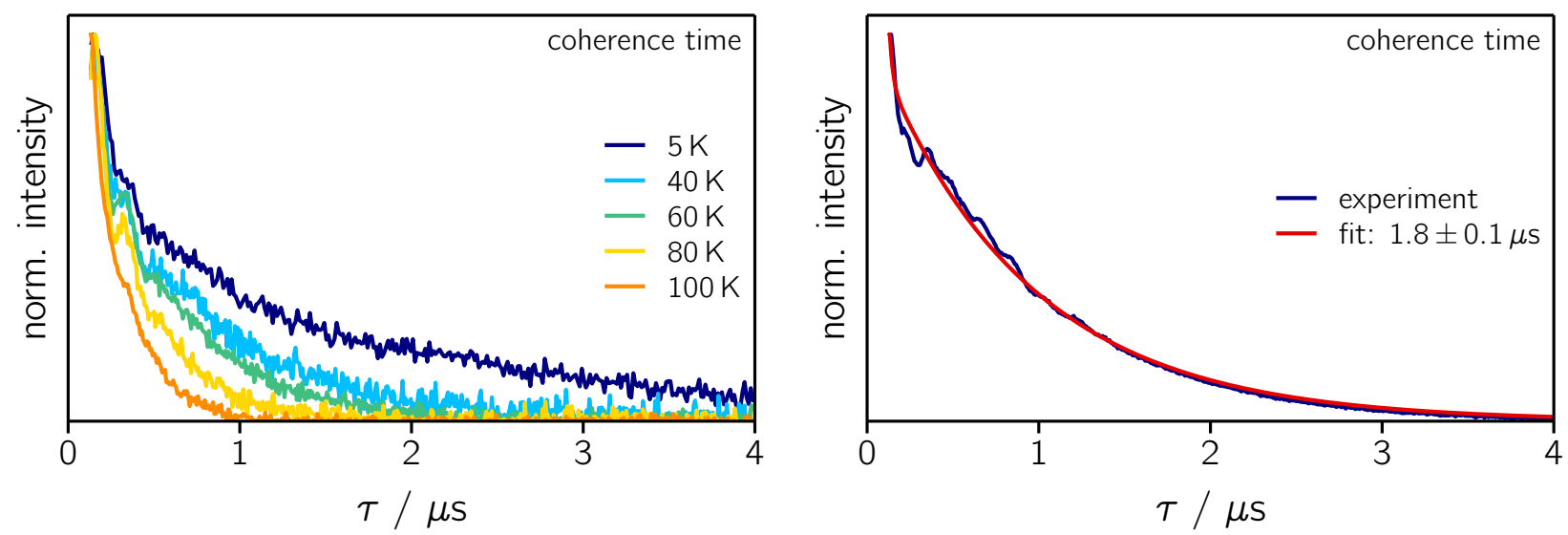

Figure S15: Measurement of the quartet state coherence times of PDI-TEMPO in frozen toluene at a magnetic field position corresponding to the $\left| \pm \frac{1}{2}\right\rangle \leftrightarrow\left| \pm \frac{3}{2}\right\rangle$ transition at different temperatures (left) and fit to the coherence data in the center of the spectrum $\left(\left|+\frac{1}{2}\right\rangle \leftrightarrow\left|-\frac{1}{2}\right\rangle\right.$ transition) at $80 \mathrm{~K}$, as discussed in the main text.
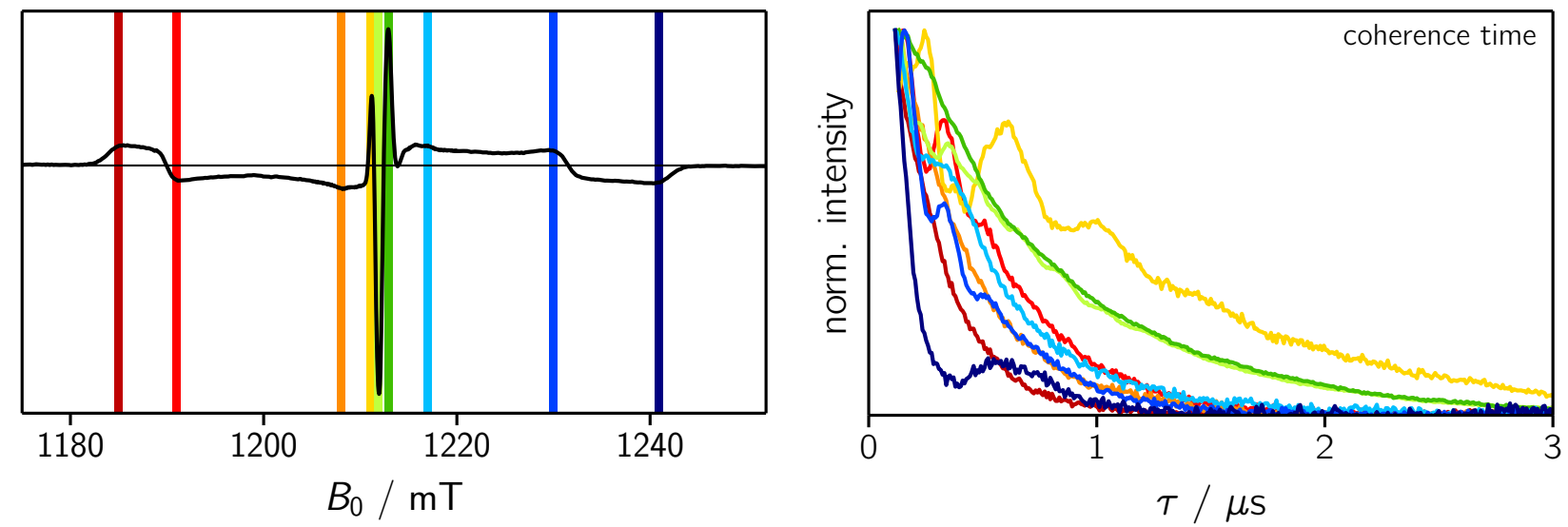

Figure S16: Measurement of the quartet state coherence times of PDI-TEMPO in frozen toluene measured at $80 \mathrm{~K}$ at different magnetic field positions (as indicated by the colored vertical lines in the corresponding quartet state spectrum shown on the left).

The spin-lattice relaxation time of the quartet state was also measured at $80 \mathrm{~K}$ using an inversion recovery sequence. A monoexponential fit of the trace measured at $1212 \mathrm{mT}$ yielded a $T_{1}$ of $130 \mu \mathrm{s}$.

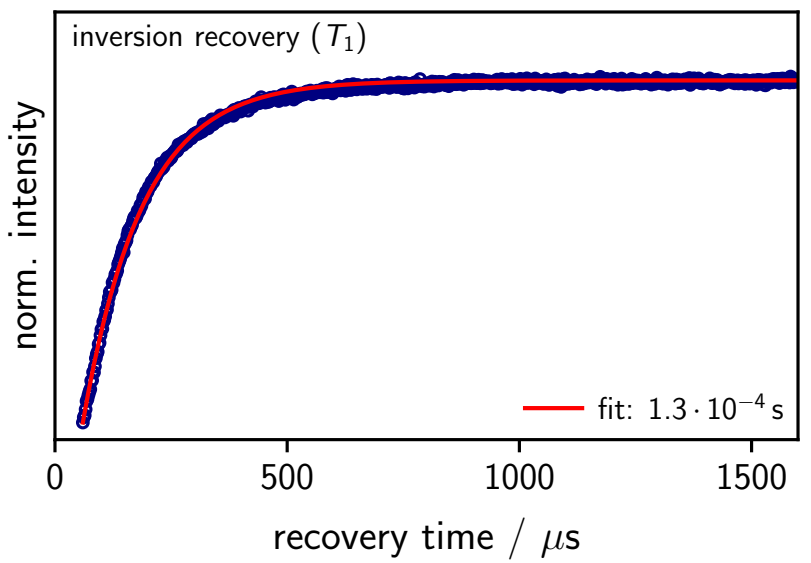

Figure S17: Measurement of the spin lattice relaxation time of the quartet state of PDI-TEMPO in frozen toluene at $80 \mathrm{~K}$ at a field position corresponding to the $\left|+\frac{1}{2}\right\rangle \leftrightarrow\left|-\frac{1}{2}\right\rangle$ transition (1212 $\mathrm{mT}$ at $34.0 \mathrm{GHz}$ ). 


\section{Rabi oscillations}

The Fourier transform of the Rabi oscillations, shown in the main text, was computed after background correction using a polynomial background function, windowing, and zero-filling to 2048 data points. The frequency spectra are shown in Figure S18 for three different microwave attenuations of 5,8 and $11 \mathrm{~dB}$ and show peaks at $18.9,13.4$ and $9.5 \mathrm{MHz}$, respectively. A change in the microwave power of $3 \mathrm{~dB}$ should lead to a change in the frequency of $\sqrt{2}$, which agrees very well with the experimental results.

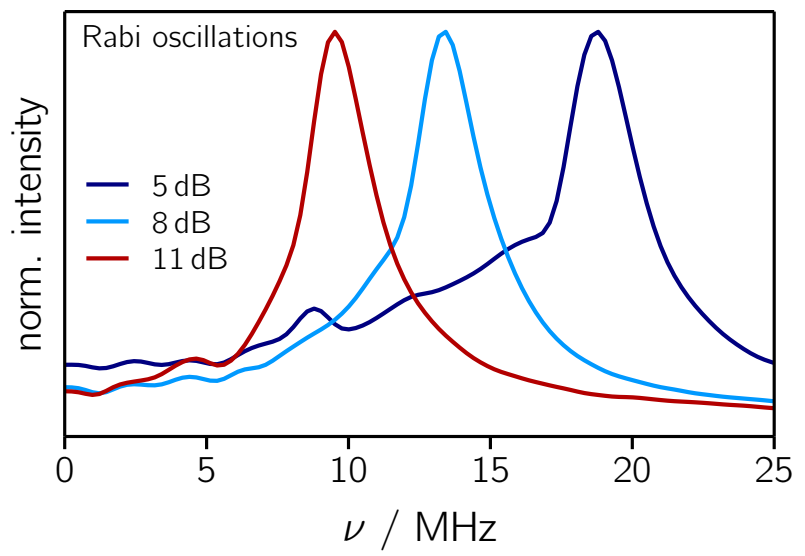

Figure S18: Fourier transform of the Rabi oscillation traces (as shown in the main text) acquired for the quartet state of PDI-TEMPO at $80 \mathrm{~K}$.

The same experiment on the quartet state of PDI-TEMPO was also performed at $60 \mathrm{~K}$ at a field position corresponding to the $\left|+\frac{1}{2}\right\rangle \leftrightarrow\left|-\frac{1}{2}\right\rangle$ transition and the data is shown in Figure S19. At this field position, a $T_{\mathrm{m}}$ value of $2.0 \mu \mathrm{s}$ was measured and Rabi oscillation frequencies of $20.5,10.3$ and $14.4 \mathrm{MHz}$ were obtained at microwave attenuations of 4,7 , and $10 \mathrm{~dB}$, respectively. Consequently, the figure of merit amounts to 80,58 , and 41 at these attenuations.
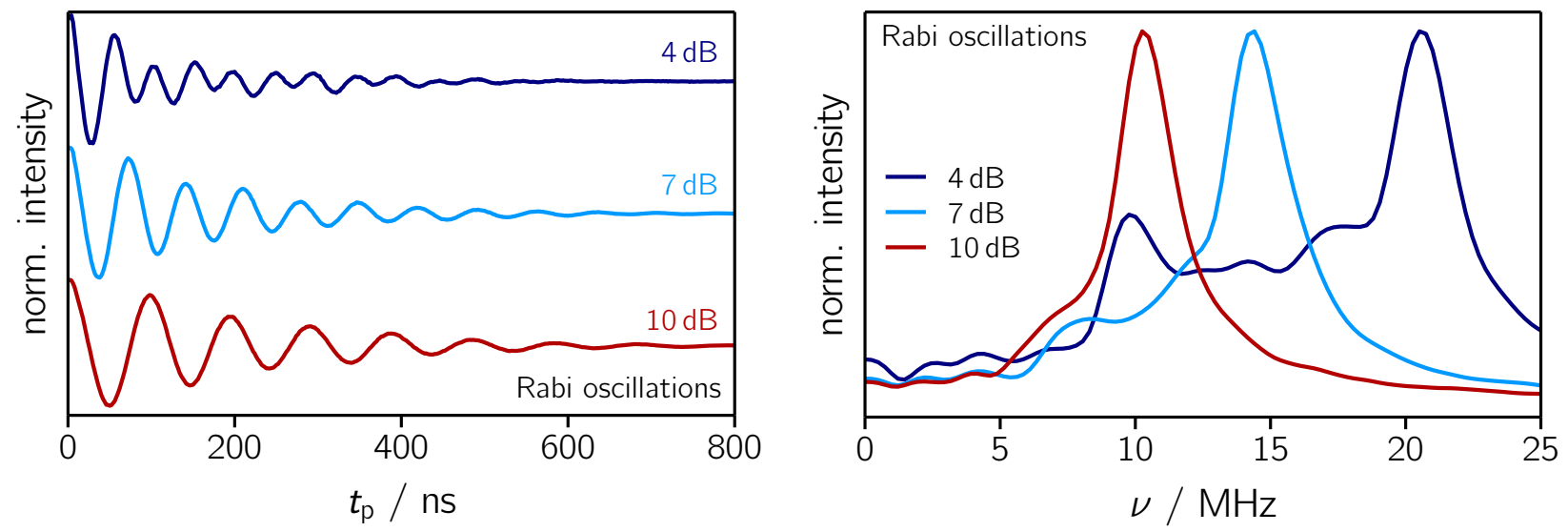

Figure S19: Rabi oscillations measured for the quartet state of PDI-TEMPO at a field position corresponding to the $\left|+\frac{1}{2}\right\rangle \leftrightarrow\left|-\frac{1}{2}\right\rangle$ transition in frozen toluene at $60 \mathrm{~K}$ (left) and corresponding Fourier transform (right).

To verify whether all quartet transitions can be manipulated coherently, Rabi oscillation experiments at $80 \mathrm{~K}$ were also performed at different magnetic field positions within the quartet spectrum. The results obtained for a microwave attenuation of $7 \mathrm{~dB}$ are shown in Figure S20 and correspond to frequencies between 11.5 and $13.4 \mathrm{MHz}$. 

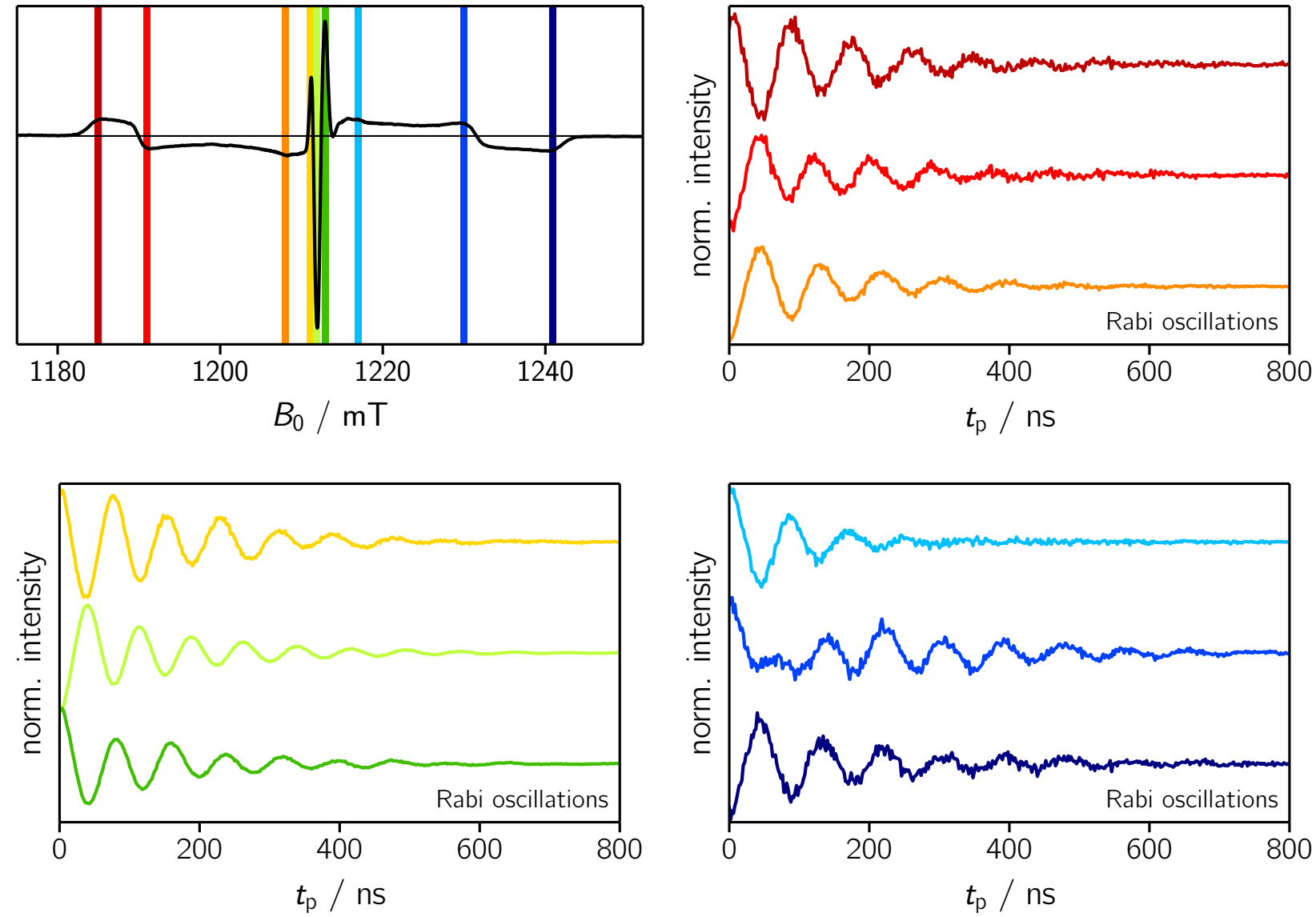

Figure S20: Rabi oscillations measured at $80 \mathrm{~K}$ for the quartet state of PDI-TEMPO at different magnetic field positions within the quartet state spectrum as indicated by colored vertical lines in the field-swept echodetected spectrum (top, left). 


\section{Effect of temperature on the spectrum}

To study the effect of the measurement temperature on the spectral shape and the signal strength (i.e. quartet state generation efficiency), quartet spectra were also recorded at different temperatures.
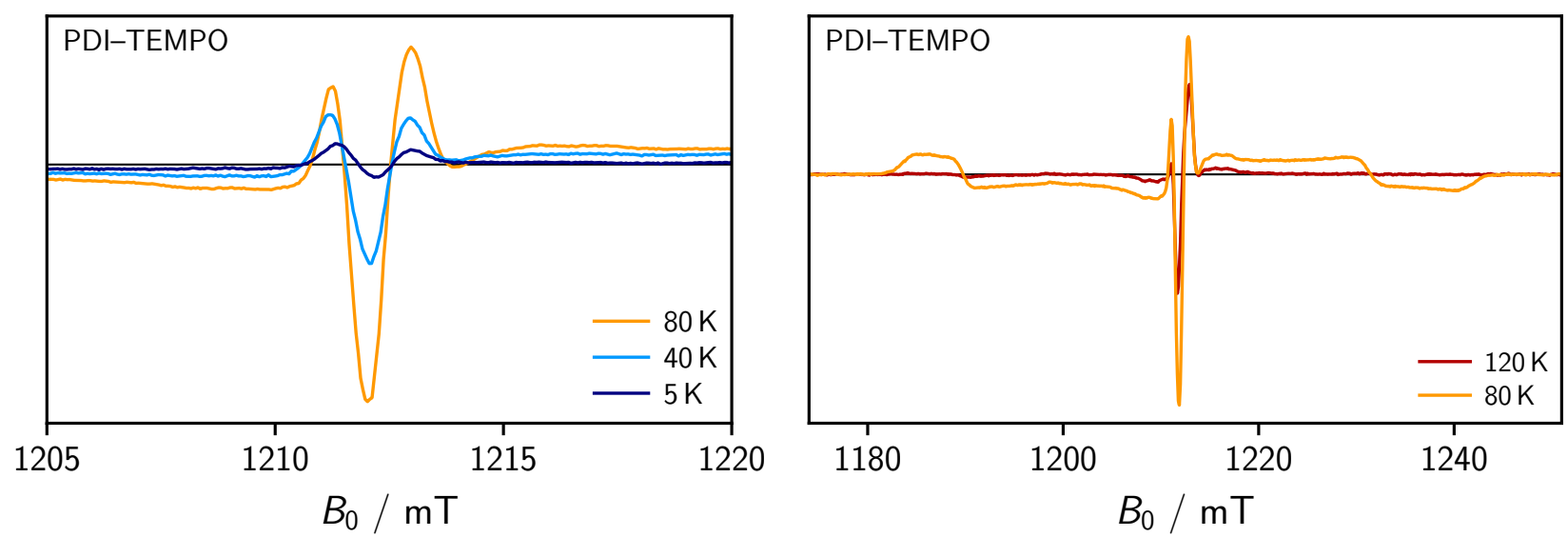

Figure S21: Comparison of the intensities of the quartet state EPR signals recorded at different temperatures.

The experimental settings were left the same, so that the signal intensities are directly comparable. As can be seen from Figure S21 the quartet state signal decreases markedly with decreasing temperature (whereas the nitroxide signal increases). Also at higher temperatures the signal intensity was found to decrease. The chosen measurement temperature of $80 \mathrm{~K}$ therefore seems to be optimal for the investigated PDI-TEMPO system.

\section{DFT calculations}

All DFT calculations were performed using the ORCA program package (version 4.0). For the calculation of the spin density, the structures were first optimized in their (doublet, triplet or quartet) ground states using the B3LYP functional in combination with the def2-TZVP basis set, RI approximation, and dispersion correction to the energies (D3). Magnetic property calculations in the doublet, triplet or quartet states used the B3LYP functional in combination with the EPR-II basis set.

A visual representation of the optimized molecular structure and calculated spin densities (iso values of 0.001) for the triplet state of PDI as well as the doublet and quartet states of PDI-TEMPO are shown in Figures S22 and S23.
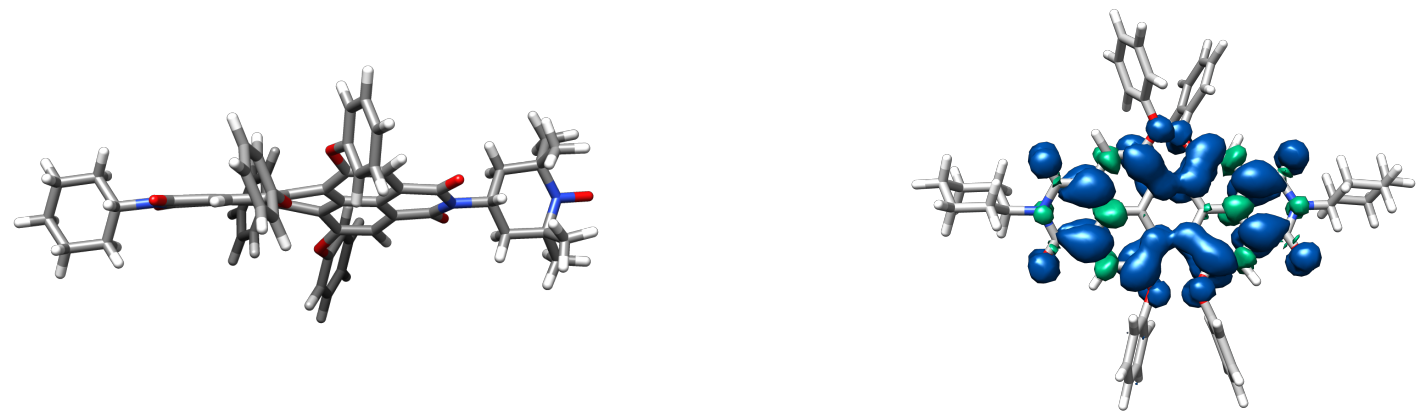

Figure S22: Visualization of the core twist of the molecular structure (left) and spin density of the excited triplet state (right) of PDI as predicted by DFT calculations. 

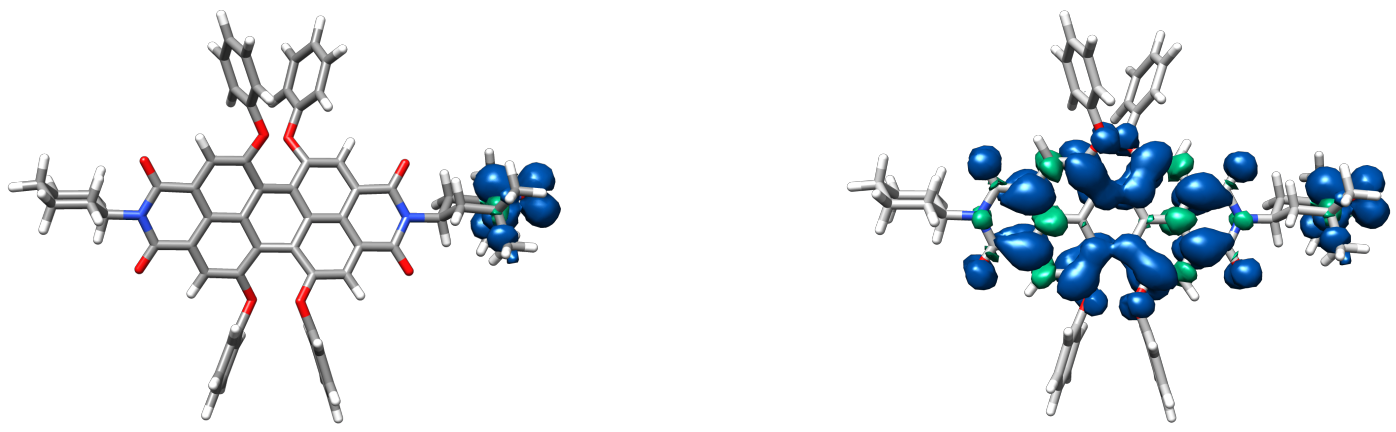

Figure S23: Visualization of the spin density in the doublet (left) and quartet (right) state of PDI-TEMPO as predicted by DFT calculations.

From the optimized structure, a center-to-center distance between PDI and TEMPO of $\sim 1.0 \mathrm{~nm}$ was obtained. However, the effective coupling distance between the two spin centres is predicted to be considerably shorter than the center-to-center distance, due to a significant delocalization of both the radical and triplet spin densities.

Tables S1 and S2 give an overview of the calculated $g$ - and $D$-values (where applicable) as well as hyperfine tensors for the triplet state of PDI and the doublet and quartet states of PDI-TEMPO. While it is known that the magnitudes of the calculated values can deviate substantially from the experimental ones (especially considering the $D$-values), the trends are typically correctly predicted. The calculations predict $g_{\text {iso }}$ values of $2.0069,2.0038$, and 2.0048 for the doublet, triplet and quartet states, respectively. The $D$-value of the triplet state is predicted to be roughly twice as large compared to $D_{\mathrm{Q}}$, meaning that the spectra of the triplet and quartet states would be expected to have a similar width. However, according to the calculations, the sign of $D_{\mathrm{Q}}$ should be negative, i.e. opposite to that of $D_{\mathrm{T}}$.

Table S1: Overview of the calculated $\mathbf{g}$-tensors for the triplet state of PDI and doublet and quartet states of PDI-TEMPO.

\begin{tabular}{|c|c|c|c|}
\hline Compound & $g_{T}$ & $g_{\mathrm{R}}$ & $g_{\mathrm{Q}}$ \\
\hline PDI & {$\left[\begin{array}{lll}2.0043 & 2.0043 & 2.0027\end{array}\right]$} & - & - \\
\hline PDI-TEMPO & - & {$\left[\begin{array}{lll}2.0095 & 2.0085 & 2.0028\end{array}\right]$} & [2.0061 2.0045 2.0039] \\
\hline
\end{tabular}

Table S2: Overview of the calculated $D$-values for the triplet state of PDI and quartet state of PDI-TEMPO as well as the nitrogen hyperfine couplings (of the nitroxide) in the doublet and quartet states of PDI-TEMPO.

\begin{tabular}{|c|c|c|c|c|}
\hline Compound & $D_{\mathrm{T}} / \mathrm{MHz}$ & $D_{\mathrm{Q}} / \mathrm{MHz}$ & $A_{\mathrm{R}}^{\mathrm{N}} / \mathrm{MHz}$ & $A_{Q}^{N} / \mathrm{MHz}$ \\
\hline PDI & +464 & - & - & - \\
\hline PDI-TEMPO & - & -221 & [11.7 11.2 83.3] & {$\left[\begin{array}{llll}3.9 & 3.7 & 27.8\end{array}\right]$} \\
\hline
\end{tabular}




\section{References}

[1] Berberich, M.; Natali, M.; Spenst, P.; Chiorboli, C.; Scandola, F.; Würthner, F. Nondestructive photoluminescence read-out by intramolecular electron transfer in a perylene bisimide-diarylethene dyad. Nat. Photonics 2018, 12, 516-527.

[2] Wu, Y.; Young, R. M.; Frasconi, M.; Schneebeli, S. T.; Spenst, P.; Gardner, D. M.; Brown, K. E.; Würthner, F.; Stoddart, J. F.; Wasielewski, M. R. Ultrafast photoinduced symmetry-breaking charge separation and electron sharing in perylenediimide molecular triangles. J. Am. Chem. Soc. 2015, 137, 13236-13239.

[3] Stoll, S.; Schweiger, A. EasySpin, a comprehensive software package for spectral simulation and analysis in EPR. J. Magn. Reson. 2006, 178, 42-55. 